\title{
On the set of common fixed points of semigroups of nonlinear mappings in modular function spaces
}

\author{
Saud M Alsulami ${ }^{*}$ and Walter M Kozlowski
}

\section{"Correspondence:}

alsulami@kau.edu.sa

'Department of Mathematics, King

Abdulaziz University, P.O. Box

138381, Jeddah, 21323, Saudi Arabia

Full list of author information is

available at the end of the article

\begin{abstract}
We prove that the set of all common fixed points for a continuous nonexpansive semigroup of nonlinear mappings acting in modular function spaces can be represented as an intersection of fixed points sets of two nonexpansive mappings. This representation is then used to prove convergence of several iterative methods for construction of common fixed points of semigroups of nonlinear mappings. We also demonstrate an example how the results of this paper can be applied for constructing a stationary point of a process defined by the Urysohn integral operator. MSC: Primary 47H09; secondary 46B20; 47H10; 47H20; 47E30; 47J25

Keywords: fixed point; common fixed point; nonexpansive mapping; semigroup of nonlinear mappings; modular function space; Orlicz space; convex modular; fixed point iteration process; Mann process; Ishikawa process
\end{abstract}

\section{Introduction}

The purpose of this paper is to prove that the set of all common fixed points for a continuous nonexpansive semigroup of nonlinear mappings acting in modular function spaces can be represented as an intersection of fixed point sets of two nonexpansive mappings, where nonexpansiveness is understood in the modular sense. Modular function spaces are natural generalizations of both function and sequence variants of many important, from applications perspective, spaces like Lebesgue, Orlicz, Musielak-Orlicz, Lorentz, OrliczLorentz, Calderon-Lozanovskii spaces and many others; see $[1,2]$ for an extensive list of examples and special cases.

The fixed point theory in modular function spaces originated in the 1990 seminal paper by Khamsi, Kozlowski and Reich [3]. In that paper, the authors showed that there exist mappings which are $\rho$-nonexpansive but are not norm-nonexpansive. They demonstrated that for a mapping $T$ to be norm nonexpansive in a modular function space $L_{\rho}$, a stronger than $\rho$-nonexpansiveness assumption is needed: $\rho(\lambda(T(x)-T(x))) \leq \rho(\lambda(x-y))$ for any $\lambda \geq 0$. From this perspective, the fixed point theory in modular function spaces should be considered as complementary to the fixed point theory in normed spaces and in metric spaces. It is worthwhile to mention that from the perspective of applications, modular type conditions are typically more easily verified than their metric or norm counterparts. For earlier and recent results of fixed point theory in modular function spaces, refer, e.g., to $[2,4-13]$.

02014 Alsulami and Kozlowski; licensee Springer. This is an Open Access article distributed under the terms of the Creative Commons Attribution License (http://creativecommons.org/licenses/by/2.0), which permits unrestricted use, distribution, and reproduction in any medium, provided the original work is properly cited. 
Let us recall that a family $\left\{T_{t}\right\}_{t \geq 0}$ of mappings forms a semigroup if $T_{0}(x)=x, T_{s+t}=$ $T_{s}\left(T_{t}(x)\right)$. Such a situation is quite typical in mathematics and applications. For instance, in the theory of dynamical systems, the modular function space $L_{\rho}$ would define the state space and the mapping $(t, x) \rightarrow T_{t}(x)$ would represent the evolution function of a dynamical system. The question about the existence of common fixed points, and about the structure of the set of common fixed points, can be interpreted as a question whether there exist points that are fixed during the state space transformation $T_{t}$ at any given point of time $t$, and if yes - what the structure of a set of such points may look like. In the setting of this paper, the state space may be infinite dimensional. Therefore, it is natural to apply these results not only to deterministic dynamical systems but also to stochastic dynamical systems.

An existence of common fixed points of $\rho$-nonexpansive semigroups was demonstrated in 2011 [12]. However, a structure of the set of common fixed points can be a priori very complicated and therefore it can be difficult to apply any methods of construction of such common fixed points, which is of a major importance for applications. In the current paper, we show that in the case of a continuous nonexpansive semigroup, the set of its common fixed points can be actually represented by an intersection of fixed point sets of just two suitably chosen, nonexpansive mappings. The idea of such representation is known in Banach spaces; see, e.g., the 2005 paper by Suzuki [14] and references therein. However, the case of $\rho$-nonexpansive mappings acting in modular function spaces have not been investigated prior to the current paper. It is worthwhile to mention that we use only convexity of the function modular $\rho$ as it does not need to have any triangle inequality of homogeneity properties. This shows the strength of the convexity assumptions because convexity of $\rho$ suffices to prove both the existences and the representation of a set of common fixed points.

We use this representation to show how the Mann and Ishikawa type iterative methods can be used for the construction of common fixed points of continuous nonexpansive semigroups. The idea of using such processes in this context can be traced back to the seminal 1950s-1970s papers by Mann [15], Krasnosel'skii [16], Ishikawa [17], Reich [18, 19], and others. See also an extensive body of work from the 1980s and 1990s [20-31], and more recent research from the current century [13, 32-46] and the works referred there. We also show an example how the results of this paper can be applied for constructing a stationary point of an Urysohn process.

\section{Preliminaries}

Let us introduce basic notions related to modular function spaces and related notation which will be used in this paper. For further details, we refer the reader to preliminary sections of the recent articles $[10,11,13]$ or to the survey article [2]; see also $[1,47,48]$ for the standard framework of modular function spaces.

Let $\Omega$ be a nonempty set and $\Sigma$ be a nontrivial $\sigma$-algebra of subsets of $\Omega$. Let $\mathcal{P}$ be a $\delta$-ring of subsets of $\Omega$ such that $E \cap A \in \mathcal{P}$ for any $E \in \mathcal{P}$ and $A \in \Sigma$. Let us assume that there exists an increasing sequence of sets $K_{n} \in \mathcal{P}$ such that $\Omega=\bigcup K_{n}$. By $\mathcal{E}$ we denote the linear space of all simple functions with supports from $\mathcal{P}$. By $\mathcal{M}_{\infty}$ we denote the space of all extended measurable functions, i.e., all functions $f: \Omega \rightarrow[-\infty, \infty]$ such that there exists a sequence $\left\{g_{n}\right\} \subset \mathcal{E},\left|g_{n}\right| \leq|f|$ and $g_{n}(\omega) \rightarrow f(\omega)$ for all $\omega \in \Omega$. By $1_{A}$ we denote the characteristic function of the set $A$. 
Definition 2.1 Let $\rho: \mathcal{M}_{\infty} \rightarrow[0, \infty]$ be a nontrivial, convex and even function. We say that $\rho$ is a regular convex function pseudomodular if:

(i) $\rho(0)=0$;

(ii) $\rho$ is monotone, i.e., $|f(\omega)| \leq|g(\omega)|$ for all $\omega \in \Omega$ implies $\rho(f) \leq \rho(g)$, where $f, g \in \mathcal{M}_{\infty}$

(iii) $\rho$ is orthogonally subadditive, i.e., $\rho\left(f 1_{A \cup B}\right) \leq \rho\left(f 1_{A}\right)+\rho\left(f 1_{B}\right)$ for any $A, B \in \Sigma$ such that $A \cap B \neq \emptyset, f \in \mathcal{M}$;

(iv) $\rho$ has the Fatou property, i.e., $\left|f_{n}(\omega)\right| \uparrow|f(\omega)|$ for all $\omega \in \Omega$ implies $\rho\left(f_{n}\right) \uparrow \rho(f)$, where $f \in \mathcal{M}_{\infty}$;

(v) $\rho$ is order continuous in $\mathcal{E}$, i.e., $g_{n} \in \mathcal{E}$ and $\left|g_{n}(\omega)\right| \downarrow 0$ implies $\rho\left(g_{n}\right) \downarrow 0$.

Similarly, as in the case of measure spaces, we say that a set $A \in \Sigma$ is $\rho$-null if $\rho\left(g 1_{A}\right)=0$ for every $g \in \mathcal{E}$. We say that a property holds $\rho$-almost everywhere if the exceptional set is $\rho$-null. As usual we identify any pair of measurable sets whose symmetric difference is $\rho$-null as well as any pair of measurable functions differing only on a $\rho$-null set. With this in mind, we define $\mathcal{M}=\left\{f \in \mathcal{M}_{\infty}:|f(\omega)|<\infty \rho\right.$-a.e. $\}$, where each element is actually an equivalence class of functions equal $\rho$-a.e. rather than an individual function.

Definition 2.2 We say that a regular function pseudomodular $\rho$ is a regular convex function modular if $\rho(f)=0$ implies $f=0 \rho$-a.e. The class of all nonzero regular convex function modulars defined on $\Omega$ will be denoted by $\Re$.

Definition $2.3[1,47,48]$ Let $\rho$ be a convex function modular. A modular function space is the vector space $L_{\rho}=\{f \in \mathcal{M}: \rho(\lambda f) \rightarrow 0$ as $\lambda \rightarrow 0\}$.

The following notions will be used throughout the paper.

Definition 2.4 Let $\rho \in \Re$.

(a) We say that $\left\{f_{n}\right\}$ is $\rho$-convergent to $f$ and write $f_{n} \rightarrow f(\rho)$ if and only if $\rho\left(f_{n}-f\right) \rightarrow 0$.

(b) A sequence $\left\{f_{n}\right\}$, where $f_{n} \in L_{\rho}$, is called $\rho$-Cauchy if $\rho\left(f_{n}-f_{m}\right) \rightarrow 0$ as $n, m \rightarrow \infty$.

(c) A set $B \subset L_{\rho}$ is called $\rho$-closed if for any sequence of $f_{n} \in B$, the convergence $f_{n} \rightarrow f(\rho)$ implies that $f$ belongs to $B$.

(d) A set $B \subset L_{\rho}$ is called $\rho$-bounded if $\sup \{\rho(f-g): f \in B, g \in B\}<\infty$.

(e) A set $B \subset L_{\rho}$ is called strongly $\rho$-bounded if there exists $\beta>1$ such that $M_{\beta}(B)=\sup \{\rho(\beta(f-g)): f \in B, g \in B\}<\infty$.

Since $\rho$ fails in general the triangle identity, many of the known properties of limit may not extend to $\rho$-convergence. For example, $\rho$-convergence does not necessarily imply the $\rho$-Cauchy condition. However, it is important to remember that the $\rho$-limit is unique when it exists. The following proposition brings together a few facts that will be often used in the proofs of our results.

Proposition 2.1 Let $\rho \in \Re$.

(i) $L_{\rho}$ is $\rho$-complete.

(ii) $\rho$-balls $B_{\rho}(x, r)=\left\{y \in L_{\rho}: \rho(x-y) \leq r\right\}$ are $\rho$-closed and $\rho$-a.e. closed.

(iii) If $\rho\left(\alpha f_{n}\right) \rightarrow 0$ for an $\alpha>0$, then there exists a subsequence $\left\{g_{n}\right\}$ of $\left\{f_{n}\right\}$ such that $g_{n} \rightarrow 0 \rho$-a.e. 
(iv) $\rho(f) \leq \liminf \rho\left(f_{n}\right)$ whenever $f_{n} \rightarrow f \rho$-a.e. (Note: this property is equivalent to the Fatou property.)

We will also need the definition of the $\Delta_{2}$-property of a function modular; see, e.g., $[1,13]$.

Definition 2.5 Let $\rho \in \Re$. We say that $\rho$ has the $\Delta_{2}$-property if

$$
\sup _{n} \rho\left(2 f_{n}, D_{k}\right) \rightarrow 0
$$

whenever $D_{k} \downarrow \emptyset$ and $\sup _{n} \rho\left(f_{n}, D_{k}\right) \rightarrow 0$.

The modular equivalents of uniform convexity were introduced in [11].

Definition 2.6 Let $\rho \in \Re$. We define the following uniform convexity type properties of the function modular $\rho$ :

(i) Let $r>0, \varepsilon>0$. Define

$$
D(r, \varepsilon)=\left\{(f, g): f, g \in L_{\rho}, \rho(f) \leq r, \rho(g) \leq r, \rho(f-g) \geq \varepsilon r\right\} .
$$

Let

$$
\delta(r, \varepsilon)=\inf \left\{1-\frac{1}{r} \rho\left(\frac{f+g}{2}\right):(f, g) \in D(r, \varepsilon)\right\} \quad \text { if } D(r, \varepsilon) \neq \emptyset,
$$

and $\delta(r, \varepsilon)=1$ if $D(r, \varepsilon)=\emptyset$. We say that $\rho$ satisfies $(U C)$ if for every $r>0, \varepsilon>0$, $\delta(r, \varepsilon)>0$. Note that for every $r>0, D(r, \varepsilon) \neq \emptyset$ for $\varepsilon>0$ small enough.

(ii) We say that $\rho$ satisfies (UUC) if for every $s \geq 0, \varepsilon>0$ there exists

$$
\eta(s, \varepsilon)>0
$$

depending on $s$ and $\varepsilon$ such that

$$
\delta(r, \varepsilon)>\eta(s, \varepsilon)>0 \quad \text { for } r>s .
$$

Let us also introduce the modular definition of strict convexity following [11].

Definition 2.7 We say that $\rho$ is strictly convex $(S C)$ if $\rho(f)=\rho(g)$ and

$$
\rho(\lambda f+(1-\lambda) g)=\lambda \rho(f)+(1-\lambda) \rho(g)
$$

imply that $f=g$, where $\lambda \in(0,1)$ and $f, g \in L_{\rho}$.

Proposition 2.2 By Proposition 3.3 from [11] it follows that if $\rho$ is (UUC), then it is also $(S C)$.

Remark 2.1 The notion of a modular function space has been generalized recently to a more abstract, nonlinear case of a modular metric space; see, e.g., [49, 50]. Let us recall 
that a function $\omega:(0, \infty) \times X \times X \rightarrow[0, \infty]$ is called a convex modular metric on a set $X$ if: (i) $x=y$ if and only if $\omega_{\lambda}(x, y)=0$ for all $\lambda>0$; (ii) $\omega_{\lambda}(x, y)=\omega_{\lambda}(y, x)$ for all $\lambda>0, x \in X$, $y \in X$; (iii) $\omega_{\lambda+\mu}(x, y) \leq \frac{\lambda}{\lambda+\mu} \omega_{\lambda}(x, z)+\frac{\mu}{\lambda+\mu} \omega_{\mu}(z, y)$. In this context, given $x_{0} \in X$, the modular metric space around $x_{0}$, denoted by $X_{\omega}\left(x_{0}\right)$, is defined as

$$
X_{\omega}\left(x_{0}\right)=\left\{x \in X: \omega_{\lambda}\left(x, x_{0}\right) \rightarrow 0 \text { as } \lambda \rightarrow \infty\right\} .
$$

Furthermore, $X_{\omega}\left(x_{0}\right)$ can be endowed with a metric given by

$$
d_{\omega}^{*}(x, y)=\inf \left\{\lambda>0: \omega_{\lambda}(x, y) \leq 1\right\} .
$$

Given a modular function space $L_{\rho}$, where $\rho$ is a convex function modular, it is not difficult to demonstrate that the formula

$$
\omega_{\lambda}(f, g)=\rho\left(\frac{f-g}{\lambda}\right)
$$

defines a modular metric on $L_{\rho}$. Moreover, we have

$$
\|f=g\|_{\rho}=d_{\omega}^{*}(f, g)
$$

for any $f, g \in L_{\rho}$.

Let us also introduce modular definitions of Lipschitzian and nonexpansive mappings and associated definitions of semigroups of nonlinear mappings acting within a modular function space.

Definition 2.8 [12] Let $\rho \in \Re$ and let $C \subset L_{\rho}$ be nonempty and $\rho$-closed. A mapping $T: C \rightarrow C$ is called $\rho$-Lipschitzian if there exists a constant $L>0$ such that

$$
\rho(T(f)-T(g)) \leq L \rho(f-g) \quad \text { for any } f, g \in L_{\rho} .
$$

$T$ is called a $\rho$-nonexpansive mapping if $L=1$.

For any mapping $T$, by $F(T)$ we denote the set of all fixed points of $T$.

The following theorem is an immediate consequence of Theorem 4.1 in [11].

Theorem 2.1 Assume that $\rho \in \Re$ is (UUC). Let $C$ be a $\rho$-closed, $\rho$-bounded convex nonempty subset. Then any $\rho$-nonexpansive mapping $T: C \rightarrow C$ has a fixed point. Moreover, the set of all fixed points $\operatorname{Fix}(T)$ is $\rho$-closed and convex.

Definition 2.9 [12] A one-parameter family $\mathcal{F}=\left\{T_{t}: t \geq 0\right\}$ of mappings from $C$ into itself is said to be a $\rho$-Lipschitzian (resp. $\rho$-nonexpansive) semigroup on $C$ if $\mathcal{F}$ satisfies the following conditions:

(i) $T_{0}(x)=x$ for $x \in C$;

(ii) $T_{t+s}(x)=T_{t}\left(T_{s}(x)\right)$ for $x \in C$ and $t, s \geq 0$;

(iii) for each $t \geq 0, T_{t}$ is $\rho$-Lipschitzian (resp. $\rho$-nonexpansive). 
Definition 2.10 A semigroup $\mathcal{F}=\left\{T_{t}: t \geq 0\right\}$ is called strongly continuous if for every $z \in C$, the following function

$$
\Lambda_{z}(t)=\rho\left(T_{t}(z)-z\right)
$$

is continuous at every $t \in[0, \infty)$.

Definition 2.11 A semigroup $\mathcal{F}=\left\{T_{t}: t \geq 0\right\}$ is called continuous if for every $z \in C$, the mapping $t \longmapsto T_{t}(z)$ is $\rho$-continuous at every $t \in[0, \infty)$, i.e., $\rho\left(T_{t_{n}}(z)-T_{t}(z)\right) \rightarrow 0$ as $t_{n} \rightarrow t$.

By $F(\mathcal{F})$ we denote the set of common fixed points of the semigroup $\mathcal{F}$.

Let us finish this section with the existence theorem for semigroups of nonexpansive mappings acting in modular function spaces.

Theorem 2.2 [12] Assume that $\rho \in \Re$ is (UUC). Let $C$ be a $\rho$-closed $\rho$-bounded convex nonempty subset. Let $\mathcal{F}$ be a nonexpansive semigroup on $C$. Then the set $F(\mathcal{F})$ of common fixed points is nonempty, $\rho$-closed and convex.

\section{Representation theorems}

Let us start with the following result which relates to Bruck's theorem in Banach spaces, see [51].

Theorem 3.1 Let $\rho \in \Re$ be a strictly convex function modular. Let $C \subset L_{\rho}$ and let $T$ and $S$ be two $\rho$-nonexpansive mappings from $C$ into $X$ with a common fixed point. Then, for each $\lambda \in(0,1)$, a mapping $U: C \rightarrow X$ defined by $U(x)=\lambda S(x)+(1-\lambda) T(x)$ for $x \in X$ is $\rho$-nonexpansive and $F(U)=F(S) \cap F(T)$.

Proof A straightforward calculation shows that the mapping $U$ is $\rho$-nonexpansive. It is also clear that $F(S) \cap F(T) \subset F(U)$. Therefore, to complete the proof, we need only to prove the converse inclusion.

To this end, let us fix $x \in F(U)$ and $w \in F(S) \cap F(T)$. Let us calculate:

$$
\begin{aligned}
\rho(x-w) & =\rho(\lambda S(x)+(1-\lambda) T(x)-w) \\
& \leq \lambda \rho(S(x)-w)+(1-\lambda) \rho(T(x)-w) \\
& =\lambda \rho(S(x)-S(w))+(1-\lambda) \rho(T(x)-T(w)) \\
& \leq \lambda \rho(x-w)+(1-\lambda) \rho(x-w) .
\end{aligned}
$$

In particular, (3.1) yields the following

$$
\rho(x-w)=\rho(S(x)-w)=\rho(T(x)-w) .
$$

Indeed, let us assume to the contrary that

$$
\rho(S(x)-w)<\rho(x-w)
$$


Combining (3.1) with (3.3), we have

$$
\begin{aligned}
\rho(x-w) & \leq \lambda \rho(S(x)-w)+(1-\lambda) \rho(T(x)-w) \\
& <\lambda \rho(x-w)+(1-\lambda) \rho(x-w)=\rho(x-w),
\end{aligned}
$$

which is impossible. Since the same reasoning can be applied assuming that $\rho(T(x)-w)<$ $\rho(x-w)$, we conclude that the claim (3.2) holds. Set $f=S(x)-w$ and $g=T(x)-w$ and observe that (3.2) implies that $\rho(f)=\rho(g)$. Straight calculation shows that

$$
\rho(\lambda f+(1-\lambda) g)=\rho(U(x)-w) .
$$

On the other hand, it follows from (3.2) and from the assumption $x \in F(U)$ that

$$
\begin{aligned}
\lambda \rho(f)+(1-\lambda) \rho(g) & =\lambda \rho(S(x)-w)+(1-\lambda) \rho(T(x)-w) \\
& =\lambda \rho(x-w)+(1-\lambda) \rho(x-w)=\rho(x-w)=\rho(U(x)-w) .
\end{aligned}
$$

Comparing (3.5) to (3.6), we obtain immediately

$$
\rho(\lambda f+(1-\lambda) g)=\lambda \rho(f)+(1-\lambda) \rho(g)
$$

which by the strict convexity of $\rho$ implies that $f=g$, and consequently that $S(x)=T(x)$. Compute

$$
x=U(x)=\lambda S(x)+(1-\lambda) T(x)=\lambda S(x)+(1-\lambda) S(x)=S(x),
$$

and hence $x \in F(S)$. Similarly, we can prove that $x \in F(T)$. Hence, $x \in F(S) \cap F(T)$ as claimed.

Theorem 3.2 Let $\rho \in \Re$ and let $\mathcal{F}=\left\{T_{t}: t \geq 0\right\}$ be a continuous semigroup of mappings on a subset $C$ of $L_{\rho}$. Let $\left\{\alpha_{n}\right\}$ be a sequence of nonnegative numbers converging to $\alpha \in[0, \infty)$ such that $\alpha_{n} \neq \alpha$ for all $n \in \mathbb{N}$. Then the following representation of the set of all common fixed points of $\mathcal{F}$ holds

$$
F(\mathcal{F})=\bigcap_{n=1}^{\infty} F\left(T_{\alpha_{n}}\right) .
$$

Proof We only need to prove that $\bigcap_{n=1}^{\infty} F\left(T_{\alpha_{n}}\right) \subset F(\mathcal{F})$ as the other direction is trivial. Let $z \in C$ be such that $T_{\alpha_{n}}(z)=z$ for every $n \in \mathbb{N}$.

Observe first that if $\left\{t_{n}\right\}$ is a sequence of nonnegative real numbers such that $T_{t_{n}}(z)=z$ and $t_{n} \rightarrow t$, where $t \in[0, \infty)$, then $T_{t}(z)=z$. Indeed,

$$
\begin{aligned}
\rho\left(\frac{T_{t}(z)-z}{2}\right) & \leq \frac{1}{2} \rho\left(T_{t}(z)-T_{t_{n}}(z)\right)+\frac{1}{2} \rho\left(T_{t_{n}}(z)-z\right) \\
& =\frac{1}{2} \rho\left(T_{t}(z)-T_{t_{n}}(z)\right) \rightarrow 0,
\end{aligned}
$$

by the continuity of $\mathcal{F}$. Hence $T_{t}(z)=z$ as claimed. 
The above observation implies in particular that $T_{\alpha}(z)=z$. Let us define $\beta_{n}=\left|\alpha_{n}-\alpha\right|>0$, where $n \in \mathbb{N}$. From assumptions it follows that each $\beta_{n}$ is a positive real number and that $\beta_{n} \rightarrow 0$. Note that

$$
\max \left\{\alpha_{n}, \alpha\right\}=\min \left\{\alpha_{n}, \alpha\right\}+\beta_{n} .
$$

Hence, denoting $m_{n}=\min \left\{\alpha_{n}, \alpha\right\}$ and $M_{n}=\max \left\{\alpha_{n}, \alpha\right\}$, we have

$$
T_{\beta_{n}}(z)=T_{\beta_{n}} \circ T_{m_{n}}(z)=T_{\beta_{n}+m_{n}}(z)=T_{M_{n}}(z)=z .
$$

Fix any $t>0$. By Lemma 2 in [14], there exists a sequence $\left\{k_{n}\right\}$ in $\mathbb{N} \cup\{0\}$ such that

$$
t=\sum_{i=1}^{\infty} k_{i} \beta_{i} .
$$

Denoting

$$
s_{n}=\sum_{i=1}^{n} k_{i} \beta_{i},
$$

we obtain for each $n \in \mathbb{N}$ with $s_{n}>0$

$$
T_{s_{n}}(z)=T_{\beta_{n}}^{k_{n}} \circ T_{\beta_{n-1}}^{k_{n-1}} \circ \cdots \circ T_{\beta_{2}}^{k_{2}} \circ T_{\beta_{1}}^{k_{1}}(z)=z .
$$

Since $T_{0}(z)=z$, it follows that $T_{s_{n}}(z)=z$ for every $n \in \mathbb{N}$. Because $s_{n} \rightarrow t$ and $\mathcal{F}$ is a continuous semigroup, we conclude, as previously observed, that $T_{t}(z)=z$ which concludes the proof of the theorem.

The following technical result about real numbers (Lemma 3 in [14]) will be used in the proof of our next representation theorem.

Lemma 3.1 [14] Let $\alpha$ and $\beta$ be positive real numbers satisfying $\alpha / \beta \notin \mathbb{Q}$. Define sequences $\left\{\alpha_{n}\right\}$ in $(0, \infty)$ and $\left\{k_{n}\right\}$ in $\mathbb{N}$ as follows:

(i) $\alpha_{1}=\max \{\alpha, \beta\}$;

(ii) $\alpha_{2}=\min \{\alpha, \beta\}$;

(iii) $k_{n}=\left[\alpha_{n} / \alpha_{n+1}\right]$ for all $n \in \mathbb{N}$;

(iv) $\alpha_{n+2}=\alpha_{n}-k_{n} \alpha_{n+1}$ for all $n \in \mathbb{N}$.

Then the following hold:

(a) $0<\alpha_{n+1}<\alpha_{n}$ for all $n \in \mathbb{N}$;

(b) $k_{n} \in \mathbb{N}$ for all $n \in \mathbb{N}$;

(c) $\alpha_{n} / \alpha_{n+1} \notin \mathbb{Q}$ for all $n \in \mathbb{N}$;

(d) $\left\{\alpha_{n}\right\}$ converges to 0 .

Theorem 3.3 Let $\rho \in \mathfrak{R}$ and let $\mathcal{F}=\left\{T_{t}: t \geq 0\right\}$ be a continuous semigroup of mappings on a subset $C$ of $L_{\rho}$. Let $\alpha>0$ and $\beta>0$ be two real numbers such that $\alpha / \beta \notin \mathbb{Q}$. Then

$$
F(\mathcal{F})=F\left(T_{\alpha}\right) \cap F\left(T_{\beta}\right)
$$


Proof We only need to prove that

$$
F\left(T_{\alpha}\right) \cap F\left(T_{\beta}\right) \subset F(\mathcal{F})
$$

as the converse inclusion is obvious. To this end, let us fix $z \in C$ be such that $z \in F\left(T_{\alpha}\right) \cap$ $F\left(T_{\beta}\right)$. Let $\left\{\alpha_{n}\right\}$ in $(0, \infty)$ and $\left\{k_{n}\right\}$ in $\mathbb{N}$ be two sequences defined as in Lemma 3 in [14]. We will show that

$$
T_{\alpha_{n}}(z)=T_{\alpha_{n+1}}(z)=z
$$

for every $n \in \mathbb{N}$,

$$
T_{\alpha_{1}}(z)=z \quad \text { and } \quad T_{\alpha_{2}}(z)=z
$$

Thus, equation (3.18) holds for $n=1$.

Now suppose that $T_{\alpha_{n}}(z)=T_{\alpha_{n+1}}(z)=z$. Then we have

$$
T_{\alpha_{n+2}}(z)=T_{\alpha_{n+2}} \circ T_{\alpha_{n+1}}^{k_{n}}(z)=T_{\alpha_{n+2}+k_{n} \alpha_{n+1}}(z)=T_{\alpha_{n}}(z)=T_{\alpha_{n+1}}(z)=z .
$$

Hence, by induction, $T_{\alpha_{n}}(z)=z$ for every $n \in \mathbb{N}$ and consequently,

$$
F\left(T_{\alpha}\right) \cap F\left(T_{\beta}\right) \subset \bigcap_{n=1}^{\infty} F\left(T_{\alpha_{n}}\right) .
$$

Since, by construction, $\left\{\alpha_{n}\right\}$ is a sequence of positive numbers converging to $0 \in[0, \infty)$, it follows from Theorem 3.2 that

$$
F(\mathcal{F})=\bigcap_{n=1}^{\infty} F\left(T_{\alpha_{n}}\right) .
$$

Combining (3.21) with (3.22), we obtain the desired inclusion (3.17) which completes the proof.

The next result is an immediate consequence of the fact that the uniform convexity (UUC) implies the strict convexity (SC) of $\rho$ (Proposition 2.2), and of Theorems 2.2, 3.1 and 3.3.

Theorem 3.4 Let $\rho \in \Re$ be (UUC), and let $\mathcal{F}=\left\{T_{t}: t \geq 0\right\}$ be a continuous semigroup of $\rho$-nonexpansive mappings on a $\rho$-closed, $\rho$-bounded, convex, nonempty subset of $L_{\rho}$. Let $\alpha>0$ and $\beta>0$ be two real numbers such that $\alpha / \beta \notin \mathbb{Q}$. Fix an arbitrary $\lambda \in(0,1)$. Then

$$
F(\mathcal{F})=F\left(\lambda T_{\alpha}+(1-\lambda) T_{\beta}\right)
$$

Remark 3.1 Using different methods, the conclusion of Theorem 3.4 can be proved without the assumption of the uniform convexity but assuming instead the strong continuity of the semigroup $\mathcal{F}$, see Theorem 3.1 in [46]. 


\section{Convergence of Mann iteration processes}

We concluded the previous section with Theorem 3.4 which says that, under suitable assumptions, the set of all common fixed points of a continuous semigroup of $\rho$-nonexpansive mappings is nonempty and can be represented as the set of all fixed points of just one $\rho$-nonexpansive mapping. In this section we demonstrate how this result can be applied to the construction of such a common fixed point. This idea can be summarized as follows: using the results of the previous sections, we can reduce a problem of constructing a common fixed point for a semigroup of mappings to a problem of constructing a fixed point for just one $\rho$-nonexpansive mapping. There exist well-known algorithms for solving the latter problem using generalized Mann and Ishikawa iteration processes, see [13]. In the current section, we prove the convergence of the Mann iterative process to a common fixed point of a continuous semigroup. Let us start with the definition of the Mann process, see [15].

Definition 4.1 Let $\rho \in \Re, C \subset L_{\rho}$, and let $T$ be a $\rho$-nonexpansive self-mapping on $C$. Let $\sigma \in(0,1)$. The Mann iteration process generated by the mapping $T$ and the constant $\sigma$, denoted by $M(T, \sigma)$, is defined by the following iterative formula:

$$
x_{k+1}=\sigma T\left(x_{k}\right)+(1-\sigma) x_{k}, \quad \text { where } x_{1} \in C \text { is chosen arbitrarily. }
$$

We will need the following technical results.

Lemma 4.1 [11,13] Let $\rho \in \Re$ be $(U U C)$ and let $\sigma \in(0,1)$. If there exists $R>0$ such that

$$
\begin{aligned}
& \limsup _{n \rightarrow \infty} \rho\left(f_{n}\right) \leq R, \quad \limsup _{n \rightarrow \infty} \rho\left(g_{n}\right) \leq R, \\
& \lim _{n \rightarrow \infty} \rho\left(\sigma f_{n}+(1-\sigma) g_{n}\right)=R,
\end{aligned}
$$

then

$$
\lim _{n \rightarrow \infty} \rho\left(f_{n}-g_{n}\right)=0 .
$$

Lemma 4.2 Let $\rho \in \Re$ be (UUC), $C \subset L_{\rho}$ be a $\rho$-closed, $\rho$-bounded and convex set. Let $T: C \rightarrow C$ be $\rho$-nonexpansive, and let $\sigma \in(0,1)$. Denote by $\left\{x_{k}\right\}$ a sequence of elements of $C$ generated by a Mann process $M(T, \sigma)$. Assume that $w$ is a fixed point of $T$. Then there exists $r \in \mathbb{R}$ such that

$$
\lim _{k \rightarrow \infty} \rho\left(x_{k}-w\right)=r
$$

Proof Since

$$
\begin{aligned}
\rho\left(x_{k+1}-w\right) & \leq \sigma \rho\left(T\left(x_{k}\right)-w\right)+(1-\sigma) \rho\left(x_{k}-w\right) \\
& =\sigma \rho\left(T\left(x_{k}\right)-T(w)\right)+(1-\sigma) \rho\left(x_{k}-w\right) \\
& \leq \sigma \rho\left(x_{k}-w\right)+(1-\sigma) \rho\left(x_{k}-w\right) \\
& =\rho\left(x_{k}-w\right),
\end{aligned}
$$


it follows that $\left\{x_{k}\right\}$ is a nonincreasing sequence of nonnegative numbers hence it is convergent to a number $r \in \mathbb{R}$.

Lemma 4.3 Let $\rho \in \Re$ be (UUC), $C \subset L_{\rho}$ be a $\rho$-closed, $\rho$-bounded and convex set. Let $T: C \rightarrow C$ be $\rho$-nonexpansive, and let $\sigma \in(0,1)$. Denote by $\left\{x_{k}\right\}$ a sequence of elements of $C$ generated by a Mann process $M(T, \sigma)$. Then

$$
\lim _{k \rightarrow \infty} \rho\left(T\left(x_{k}\right)-x_{k}\right)=0
$$

and

$$
\lim _{k \rightarrow \infty} \rho\left(x_{k+1}-x_{k}\right)=0 .
$$

Proof By Theorem 2.1, $T$ has at least one fixed point $w \in C$. In view of Lemma 4.2, there exists $r \in \mathbb{R}$ such that

$$
\lim _{k \rightarrow \infty} \rho\left(x_{k}-w\right)=r
$$

Note that

$$
\limsup _{k \rightarrow \infty} \rho\left(T\left(x_{k}\right)-w\right)=\limsup _{k \rightarrow \infty} \rho\left(T\left(x_{k}\right)-T(w)\right) \leq \limsup _{k \rightarrow \infty} \rho\left(x_{k}-w\right) \leq r,
$$

and that

$$
\lim _{k \rightarrow \infty} \rho\left(\sigma\left(T\left(x_{k}\right)-w\right)+(1-\sigma)\left(x_{k}-w\right)\right)=\lim _{k \rightarrow \infty} \rho\left(x_{k+1}-w\right)=r .
$$

Set $f_{k}=T\left(x_{k}\right)-w, g_{k}=x_{k}-w$, and note that $\limsup _{k \rightarrow \infty} \rho\left(g_{k}\right) \leq r$ by (4.7), and $\limsup _{k \rightarrow \infty} \rho\left(f_{k}\right) \leq r$ by (4.8). Observe also that

$$
\begin{aligned}
\lim _{k \rightarrow \infty} \rho\left(\sigma f_{k}+(1-\sigma) g_{k}\right) & =\lim _{k \rightarrow \infty} \rho\left(\sigma T\left(x_{k}\right)+(1-\sigma) x_{k}-w\right) \\
& =\lim _{k \rightarrow \infty} \rho\left(x_{k+1}-w\right)=r .
\end{aligned}
$$

Hence, it follows from Lemma 4.1 that

$$
\lim _{k \rightarrow \infty} \rho\left(T\left(x_{k}\right)-x_{k}\right)=\lim _{k \rightarrow \infty} \rho\left(f_{k}-g_{k}\right)=0,
$$

which by the construction of the sequence $\left\{x_{k}\right\}$ is equivalent to

$$
\lim _{k \rightarrow \infty} \rho\left(x_{k+1}-x_{k}\right)=0
$$

as claimed.

Remark 4.1 Please note that Lemma 4.2 and Lemma 4.3 are special cases of analogous but more general results obtained for asymptotic pointwise nonexpansive mappings, see Lemma 5.2 and Lemma 5.3 in [13]. Since the proofs for the $\rho$-nonexpansive mappings are much simpler, the authors decided to include them in the current paper for the sake of clarity and completeness. 
Let us recall now the definition of the Opial property and the strong Opial property in modular function spaces $[10,52]$.

Definition 4.2 We say that $L_{\rho}$ satisfies the $\rho$-a.e. Opial property if for every $\left\{f_{n}\right\} \in L_{\rho}$ which is $\rho$-a.e. convergent to 0 such that there exists $\beta>1$ for which

$$
\sup _{n}\left\{\rho\left(\beta f_{n}\right)\right\}<\infty
$$

the following inequality holds for any $g \in E_{\rho}$ not equal to 0

$$
\liminf _{n \rightarrow \infty} \rho\left(f_{n}\right) \leq \liminf _{n \rightarrow \infty} \rho\left(f_{n}+g\right) .
$$

Definition 4.3 We say that $L_{\rho}$ satisfies the $\rho$-a.e. strong Opial property if for every $\left\{f_{n}\right\} \in$ $L_{\rho}$ which is $\rho$-a.e. convergent to 0 such that there exists $\beta>1$ for which

$$
\sup _{n}\left\{\rho\left(\beta f_{n}\right)\right\}<\infty
$$

the following equality holds for any $g \in E_{\rho}$

$$
\liminf _{n \rightarrow \infty} \rho\left(f_{n}+g\right)=\liminf _{n \rightarrow \infty} \rho\left(f_{n}\right)+\rho(g) .
$$

Remark 4.2 Note that the $\rho$-a.e. strong Opial property implies the $\rho$-a.e. Opial property [52].

Remark 4.3 Also note that, by virtue of Theorem 2.1 in [52], every convex, orthogonally additive function modular $\rho$ has the $\rho$-a.e. strong Opial property. Let us recall that $\rho$ is called orthogonally additive if $\rho(f, A \cup B)=\rho(f, A)+\rho(f, B)$ whenever $A \cap B=\emptyset$. Therefore, all Orlicz and Musielak-Orlicz spaces must have the strong Opial property.

Note that the Opial property in the norm sense does not necessarily hold for several classical Banach function spaces. For instance, the norm Opial property does not hold for $L^{p}$ spaces for $1 \leq p \neq 2$, while the modular strong Opial property holds in $L^{p}$ for all $p \geq 1$.

The version of the demiclosedness principle we use in this paper requires the uniform continuity of the function modular $\rho$ in the sense of the following definition (see, e.g., [10]).

Definition 4.4 We say that $\rho \in \Re$ is uniformly continuous if for every $\varepsilon>0$ and $L>0$, there exists $\delta>0$ such that

$$
|\rho(g)-\rho(g+h)| \leq \varepsilon
$$

provided $\rho(h)<\delta$ and $\rho(g) \leq L$.

Let us mention that the uniform continuity holds for a large class of function modulars. For instance, it can be proved that in Orlicz spaces over a finite atomless measure [53] or in sequence Orlicz spaces [54], the uniform continuity of the Orlicz modular is equivalent to the $\Delta_{2}$-type condition. 
Theorem 4.1 (Demiclosedness principle [13]) Let $\rho \in \Re$. Assume that

(1) $\rho$ is (UUC),

(2) $\rho$ has the strong Opial property,

(3) $\rho$ has the $\Delta_{2}$ property and is uniformly continuous.

Let $C \subset L_{\rho}$ be nonempty, convex, strongly $\rho$-bounded and $\rho$-closed, $T: C \rightarrow C$ be $\rho$-nonexpansive, and $x \in C$. If $x_{n} \rightarrow x \rho$-a.e. and $\rho\left(T\left(x_{n}\right)-x_{n}\right) \rightarrow 0$, then $x \in F(T)$.

We are now ready to prove the following version of the Mann process convergence theorem for a single $\rho$-nonexpansive mapping.

Theorem 4.2 Let $\rho \in \Re$. Assume that

(1) $\rho$ is $(U U C)$

(2) $\rho$ has the strong Opial property,

(3) $\rho$ has the $\Delta_{2}$ property and is uniformly continuous.

Let $C \subset L_{\rho}$ be nonempty, $\rho$-a.e. compact, convex, strongly $\rho$-bounded and $\rho$-closed. Let $T: C \rightarrow C$ be $\rho$-nonexpansive and $\sigma \in(0,1)$. Denote by $\left\{x_{k}\right\}$ a sequence of elements of $C$ generated by a Mann process $M(T, \sigma)$. Then there exists $x \in F(T)$ such that $x_{n} \rightarrow x \rho$-a.e.

Proof Observe that by Theorem 2.1 the set of fixed points $F(T)$ is nonempty, convex and $\rho$-closed. By Lemma 4.3 the sequence $\left\{x_{k}\right\}$ is an approximate fixed point sequence, that is,

$$
\rho\left(T\left(x_{k}\right)-x_{k}\right) \rightarrow 0
$$

as $k \rightarrow \infty$. Consider $y, z \in C$, two $\rho$-a.e. cluster points of $\left\{x_{k}\right\}$. There exist then $\left\{y_{k}\right\},\left\{z_{k}\right\}$ subsequences of $\left\{x_{k}\right\}$ such that $y_{k} \rightarrow y \rho$-a.e., and $z_{k} \rightarrow z \rho$-a.e. By Theorem 4.1, $y \in F(T)$ and $z \in F(T)$. By Lemma 4.2, there exist $r_{y}, r_{z} \in \mathbb{R}$ such that

$$
r_{y}=\lim _{k \rightarrow \infty} \rho\left(x_{k}-y\right), \quad r_{z}=\lim _{k \rightarrow \infty} \rho\left(x_{k}-z\right) .
$$

We claim that $y=z$. Assume to the contrary that $y \neq z$. Then, by the strong Opial property, we have

$$
\begin{aligned}
r_{y} & =\liminf _{k \rightarrow \infty} \rho\left(y_{k}-y\right)<\liminf _{k \rightarrow \infty} \rho\left(y_{k}-z\right) \\
& =\liminf _{k \rightarrow \infty} \rho\left(z_{k}-z\right)<\liminf _{k \rightarrow \infty} \rho\left(z_{k}-y\right)=r_{y} .
\end{aligned}
$$

The contradiction implies that $y=z$. Therefore, $\left\{x_{k}\right\}$ has at most one $\rho$-a.e. cluster point. Since $C$ is $\rho$-a.e. compact, it follows that the sequence $\left\{x_{k}\right\}$ has exactly one $\rho$-a.e. cluster point, which means that $\rho\left(x_{k}\right) \rightarrow x \rho$-a.e. Using Theorem 4.1 again, we get $x \in F(T)$ as claimed.

Let us combine now Theorem 4.2 with Theorem 3.4 to demonstrate the convergence of an iterative algorithm to a common fixed point of a semigroup of nonlinear mappings in modular function spaces.

Theorem 4.3 Let $\rho \in \Re$. Assume that

(1) $\rho$ is $(U U C)$, 
(2) $\rho$ has the strong Opial property,

(3) $\rho$ has the $\Delta_{2}$ property and is uniformly continuous.

Let $C \subset L_{\rho}$ be nonempty, $\rho$-a.e. compact, convex, strongly $\rho$-bounded and $\rho$-closed. Let $\mathcal{F}=\left\{T_{t}: t \geq 0\right\}$ be a continuous semigroup of $\rho$-nonexpansive mappings on $C$. Assume that $\alpha>0$ and $\beta>0$ are two real numbers such that $\alpha / \beta \notin \mathbb{Q}$. Fix $\lambda, \kappa \in(0,1)$ such that $\kappa+\lambda<1$. Define a sequence $\left\{x_{n}\right\}$ in $C$ by $x_{1} \in C$ and

$$
x_{n+1}=\kappa T_{\alpha}\left(x_{n}\right)+\lambda T_{\beta}\left(x_{n}\right)+(1-\kappa-\lambda) x_{n}
$$

for natural $n \geq 2$. Then $\left\{x_{n}\right\} \rho$-a.e. converges to a common fixed point of the semigroup $\mathcal{F}$.

Proof Define a mapping $S$ by

$$
S=\frac{\kappa}{\kappa+\lambda} T_{\alpha}+\frac{\lambda}{\kappa+\lambda} T_{\beta},
$$

and observe that $S: C \rightarrow C$ is $\rho$-nonexpansive. Fix any $x_{1} \in C$ and let $\left\{x_{n}\right\}$ be generated by the Mann process $M(S, \sigma)$ where $\sigma=\kappa+\lambda$

$$
x_{n+1}=\sigma S\left(x_{n}\right)+(1-\sigma) x_{n},
$$

which is exactly the sequence defined by (4.21). By Theorem 4.2 there exists $x \in F(S)$ such that $x_{n} \rightarrow x \rho$-a.e. By Theorem 3.4 $F(\mathcal{F})=F(S)$, hence $x \in F(\mathcal{F})$. The proof is complete.

\section{Convergence of Ishikawa iteration processes}

The Ishikawa iteration process [17] is a two-step process generalization of the Mann process. From the numerical point of view, the Ishikawa iteration process provides more flexibility in defining the algorithm parameters, and hence providing a better control over the speed of convergence of the algorithm.

Definition 5.1 Let $\rho \in \Re, C \subset L_{\rho}$ and let $T$ be a $\rho$-nonexpansive self-mapping on $C$. Let $\sigma, \tau \in(0,1)$. The Ishikawa iteration process generated by the mapping $T$ and the constants $\sigma$ and $\tau$, denoted by $I(T, \sigma, \tau)$, is defined by the following iterative formula:

$$
x_{k+1}=\sigma T\left(\tau T\left(x_{k}\right)+(1-\tau) x_{k}\right)+(1-\sigma) x_{k}, \quad \text { where } x_{1} \in C \text { is chosen arbitrarily. }
$$

Lemma 5.1 Let $\rho \in \Re$ be (UUC). Let $C \subset L_{\rho}$ be a $\rho$-closed, $\rho$-bounded and convex set. Let $T: C \rightarrow C$ be $\rho$-nonexpansive, and let $\sigma, \tau \in(0,1)$. Denote by $\left\{x_{k}\right\}$ a sequence of elements of $C$ generated by the Ishikawa process $I(T, \sigma, \tau)$. Assume that $w$ is a fixed point of $T$. Then there exists $r \in \mathbb{R}$ such that $\lim _{k \rightarrow \infty} \rho\left(x_{k}-w\right)=r$.

Proof Using a similar calculation to the one used in the proof of Lemma 4.2, it is not difficult to prove that $\left\{x_{k}\right\}$ is a nonincreasing sequence of nonnegative numbers, hence it is convergent to a number $r \in \mathbb{R}$.

Lemma 5.2 Let $\rho \in \Re$ be (UUC). Let $C \subset L_{\rho}$ be a $\rho$-closed, $\rho$-bounded and convex set. Let $T: C \rightarrow C$ be $\rho$-nonexpansive, and let $\sigma, \tau \in(0,1)$. Denote by $\left\{x_{k}\right\}$ a sequence of elements 
of $C$ generated by the Ishikawa process $I(T, \sigma, \tau)$. Define

$$
y_{k}=\tau T\left(x_{k}\right)+(1-\tau) x_{k} .
$$

Then

$$
\lim _{k \rightarrow \infty} \rho\left(T\left(y_{k}\right)-x_{k}\right)=0
$$

or, equivalently,

$$
\lim _{k \rightarrow \infty} \rho\left(x_{k+1}-x_{k}\right)=0
$$

Proof By Theorem 2.1, $F(T) \neq \emptyset$. Let us fix $w \in F(T)$. By Lemma 5.1, $\lim _{k \rightarrow \infty} \rho\left(x_{k}-w\right)$ exists. Let us denote it by $r$. Since $w \in F(T), T \in \mathcal{T}_{r}(C)$ and $\lim _{k \rightarrow \infty} \rho\left(x_{k}-w\right)=r$, by Lemma 5.1 we have the following:

$$
\begin{aligned}
\limsup _{k \rightarrow \infty} \rho\left(T\left(y_{k}\right)-w\right) & =\limsup _{k \rightarrow \infty} \rho\left(T\left(y_{k}\right)-T(w)\right) \\
& \leq \limsup _{k \rightarrow \infty} \rho\left(y_{k}-w\right) \\
& =\limsup _{k \rightarrow \infty} \rho\left(\tau T\left(x_{k}\right)+(1-\tau) x_{k}-w\right) \\
& \leq \limsup _{k \rightarrow \infty}\left(\tau \rho\left(T\left(x_{k}\right)-w\right)+(1-\tau) \rho\left(x_{k}-w\right)\right) \\
& \leq \limsup _{k \rightarrow \infty}\left(\tau \rho\left(x_{k}-w\right)+(1-\tau) \rho\left(x_{k}-w\right)\right) \leq r .
\end{aligned}
$$

Note that

$$
\begin{aligned}
& \lim _{k \rightarrow \infty} \rho\left(\sigma\left(T\left(y_{k}\right)-w\right)+(1-\sigma)\left(x_{k}-w\right)\right) \\
& \quad=\lim _{k \rightarrow \infty} \rho\left(\sigma T\left(y_{k}\right)+(1-\sigma) x_{k}-w\right)=\lim _{k \rightarrow \infty} \rho\left(x_{k+1}-w\right)=r .
\end{aligned}
$$

Applying Lemma 4.1 with $u_{k}=T\left(y_{k}\right)-w$ and $v_{k}=x_{k}-w$, we obtain the desired equality $\lim _{k \rightarrow \infty} \rho\left(T\left(y_{k}\right)-x_{k}\right)=0$, while (5.4) follows from (5.3) via the construction formulas for $x_{k+1}$ and $y_{k}$.

Remark 5.1 Please note that Lemma 5.1 and Lemma 5.2 are special cases of analogous but more general results obtained for asymptotic pointwise nonexpansive mappings, see Lemma 6.2 and Lemma 6.3 in [13]. Since the proofs for the $\rho$-nonexpansive mappings are much simpler, the authors decided to include them in the current paper for the sake of clarity and completeness.

Lemma 5.3 Let $\rho \in \Re$ be (UUC) satisfying $\Delta_{2}$. Let $C \subset L_{\rho}$ be a $\rho$-closed, $\rho$-bounded and convex set. Let $T: C \rightarrow C$ be $\rho$-nonexpansive, and let $\sigma, \tau \in(0,1)$. Denote by $\left\{x_{k}\right\}$ a sequence of elements of $C$ generated by the Ishikawa process $I(T, \sigma, \tau)$. Then

$$
\lim _{k \rightarrow \infty} \rho\left(T\left(x_{k}\right)-x_{k}\right)=0 .
$$


Proof Let $y_{k}=\tau T\left(x_{k}\right)+(1-\tau) x_{k}$. Hence

$$
T\left(x_{k}\right)-x_{k}=\frac{1}{1-\tau}\left(T\left(x_{k}\right)-y_{k}\right) .
$$

Since $\tau \in(0,1)$, there exists $0<s<1$ such that $\tau \leq s$. Hence,

$$
\rho\left(T\left(x_{k}\right)-x_{k}\right)=\rho\left(\frac{1}{1-\tau}\left(T\left(x_{k}\right)-y_{k}\right)\right) \leq \rho\left(\frac{1}{1-s}\left(T\left(x_{k}\right)-y_{k}\right)\right) .
$$

The right-hand side of this inequality tends to zero because $\rho\left(T\left(x_{k}\right)-y_{k}\right) \rightarrow 0$ by Lemma 5.2 and because $\rho$ satisfies $\Delta_{2}$.

Using Lemma 5.3 instead of Lemma 4.3, and Lemma 5.2 instead of Lemma 4.2, and arguing in a similar way as in the proof of Theorem 4.2, we can obtain the following convergence result for the Ishikawa process.

Theorem 5.1 Let $\rho \in \Re$. Assume that

(1) $\rho$ is (UUC),

(2) $\rho$ has the strong Opial property,

(3) $\rho$ has the $\Delta_{2}$ property and is uniformly continuous.

Let $C \subset L_{\rho}$ be nonempty, $\rho$-a.e.compact, convex, strongly $\rho$-bounded and $\rho$-closed. Let $T$ : $C \rightarrow C$ be $\rho$-nonexpansive and $\sigma \in(0,1), \tau \in(0,1)$. Denote by $\left\{x_{k}\right\}$ a sequence of elements of $C$ generated by an Ishikawa process $I(T, \sigma, \tau)$. Then there exists $x \in F(T)$ such that $x_{n} \rightarrow x$ $\rho$-a.e.

Again, let us combine Theorem 5.1 with Theorem 3.4 to demonstrate the convergence of an Ishikawa-type, two-step iterative algorithm to a common fixed point of a semigroup of nonlinear mappings in modular function spaces. Please note that, as said before, typical implementations of the Ishikawa algorithm are convergent at a faster pace than the corresponding Mann schema.

Theorem 5.2 Let $\rho \in \Re$. Assume that

(1) $\rho$ is (UUC),

(2) $\rho$ has the strong Opial property,

(3) $\rho$ has the $\Delta_{2}$ property and is uniformly continuous.

Let $C \subset L_{\rho}$ be nonempty, $\rho$-a.e. compact, convex, strongly $\rho$-bounded and $\rho$-closed. Let $\mathcal{F}=\left\{T_{t}: t \geq 0\right\}$ be a continuous semigroup of $\rho$-nonexpansive mappings on $C$. Assume that $\alpha>0$ and $\beta>0$ are two real numbers such that $\alpha / \beta \notin \mathbb{Q}$. Fix $\lambda, \kappa \in(0,1)$ such that $\kappa+\lambda<1$. Define a sequence $\left\{x_{n}\right\}$ in $C$ by $x_{1} \in C$ and

$$
\begin{aligned}
x_{n+1}= & \kappa T_{\alpha}\left(\kappa T_{\alpha}\left(x_{n}\right)+\lambda T_{\beta}\left(x_{n}\right)+(1-\kappa-\lambda) x_{n}\right) \\
& +\lambda T_{\beta}\left(\kappa T_{\alpha}\left(x_{n}\right)+\lambda T_{\beta}\left(x_{n}\right)+(1-\kappa-\lambda) x_{n}\right)+(1-\kappa-\lambda) x_{n}
\end{aligned}
$$

for natural $n \geq 2$. Then $\left\{x_{n}\right\} \rho$-a.e. converges to a common fixed point of the semigroup $\mathcal{F}$.

Proof Similarly as in the Mann process case, let us define a mapping $S$ by

$$
S=\frac{\kappa}{\kappa+\lambda} T_{\alpha}+\frac{\lambda}{\kappa+\lambda} T_{\beta}
$$


and note that $S: C \rightarrow C$ is $\rho$-nonexpansive. Fix any $x_{1} \in C$ and let $\left\{x_{n}\right\}$ be generated by the two-step Ishikawa process $I(S, \sigma, \tau)$, where $\sigma=\kappa+\lambda$ and $\tau=\kappa+\lambda$,

$$
x_{n+1}=\sigma S\left(\tau S\left(x_{n}\right)+(1-\tau) x_{n}\right)+(1-\sigma) x_{n},
$$

which is exactly the sequence defined by (5.10). By Theorem 5.1 there exists $x \in F(S)$ such that $x_{n} \rightarrow x \rho$-a.e. By Theorem 3.4 $F(\mathcal{F})=F(S)$, hence $x \in F(\mathcal{F})$, which completes the proof.

\section{Application to construction of a stationary point of the Urysohn process}

In this section we provide an example how the results of the preceding sections can be utilized for constructing a stationary point of a process defined by the Urysohn operator

$$
T(f)(x)=\int_{0}^{1} k(x, y,|f(y)|) d y+f_{0}(x)
$$

where $f_{0}$ is a fixed function and $f:[0,1] \rightarrow \mathbb{R}$ is Lebesgue measurable. For the kernel $k$, we assume that

(a) $k:[0,1] \times[0,1] \times \mathbb{R}_{+} \rightarrow \mathbb{R}_{+}$is Lebesgue measurable,

(b) $k(x, y, 0)=0$,

(c) $k(x, y, \cdot)$ is continuous, convex and increasing to $+\infty$,

(d) $\int_{0}^{1} k(x, y, t) d x>0$ for $t>0$ and $y \in(0,1)$.

Assume in addition that for almost all $t \in[0,1]$ and for any two measurable functions $f, g$, there holds

$$
\begin{aligned}
& \int_{0}^{1}\left\{\int_{0}^{1} k(t, u,|k(u, v,|f(v)|)-k(u, v,|g(v)|)|) d v\right\} d u \\
& \quad \leq \int_{0}^{1} k(t, u,|f(u)-g(u)|) d u .
\end{aligned}
$$

Setting $\rho(f)=\int_{0}^{1}\left\{\int_{0}^{1} k(x, y,|f(y)|) d y\right\} d x$ and using Jensen's inequality, it is easy to show that $\rho$ is a convex function modular on the space of measurable functions defined in $[0,1]$, and that $\rho(T(f)-T(g)) \leq \rho(f-g)$, that is, $T$ is nonexpansive with respect to $\rho$. Let us fix $r>0$ and set $C=\left\{f \in E_{\rho}: \rho\left(f-f_{0}\right) \leq r\right\}$. It is easy to see that $T: C \rightarrow C$. If we assume additionally that there exist a constant $M>0$ and a Lebesgue-integrable function $h:[0,1] \times[0,1] \rightarrow$ $[0, \infty)$ such that for every $u \geq 0$ and $x, y \in[0,1]$,

$$
k(x, y, 2 u) \leq M k(x, y, u)+h(x, y)
$$

then the modular $\rho$ has the property $\Delta_{2}$ in the sense of Definition 2.5. It can be shown, see [55], that, given $f \in C$, the following initial value problem

$$
\left\{\begin{array}{l}
u(0)=f \\
u^{\prime}(t)+(I-T) u(t)=0
\end{array}\right.
$$

has a solution $u_{f}:[0,+\infty] \rightarrow C$. As proved by Khamsi in [5], the formula

$$
S_{t}(f)=u_{f}(t)
$$


defines the semigroup of $\rho$-nonexpansive mappings. Note that $\rho$ in this example is orthogonally additive and hence it has the strong Opial property, see [52]. Therefore, assuming $\rho$ is $(U U C)$ and uniformly continuous, see $[3,53,56]$ for several criteria, we can use our methods (Theorem 4.3 and Theorem 5.2) to construct a common fixed point of the semigroup $\left\{S_{t}\right\}$ which will be a stationary point of the Urysohn process defined by the evolution function $(t, f) \rightarrow u_{f}(t) \in C$.

\section{Competing interests}

The authors declare that they have no competing interest.

\section{Authors' contributions}

Both authors equally participated in all stages of preparations of this article. Both authors read and approved the final manuscript.

\section{Author details}

${ }^{1}$ Department of Mathematics, King Abdulaziz University, P.O. Box 138381, Jeddah, 21323, Saudi Arabia. ${ }^{2}$ School of Mathematics and Statistics, University of New South Wales, Sydney, NSW 2052, Australia.

\section{Acknowledgements}

This research was funded by the Deanship of Scientific Research (DSR), King Abdulaziz University, Jeddah, under grant No. (400/130/1433). The authors, therefore, acknowledge with thanks the DSR financial support. The authors would like to thank the referee for the valuable suggestions to improve the presentation of the paper.

\section{Received: 18 July 2013 Accepted: 4 December 2013 Published: 03 Jan 2014}

\section{References}

1. Kozlowski, WM: Modular Function Spaces. Series of Monographs and Textbooks in Pure and Applied Mathematics, vol. 122. Dekker, New York (1988)

2. Kozlowski, WM: Advancements in fixed point theory in modular function. Arab. J. Math. (2012) doi:10.1007/s40065-012-0051-0

3. Khamsi, MA, Kozlowski, WM, Reich, S: Fixed point theory in modular function spaces. Nonlinear Anal. 14, 935-953 (1990)

4. Khamsi, MA, Kozlowski, WM, Chen, S: Some geometrical properties and fixed point theorems in Orlicz spaces. J. Math. Anal. Appl. 155(2), 393-412 (1991)

5. Khamsi, MA: Nonlinear semigroups in modular function spaces. Math. Jpn. 37(2), 1-9 (1992)

6. Khamsi, MA: Fixed point theory in modular function spaces. In: Proceedings of the Workshop on Recent Advances on Metric Fixed Point Theory, Sevilla, September 1995, pp. 31-35 (1996). MR1440218(97m:46044)

7. Dominguez-Benavides, T, Khamsi, MA, Samadi, S: Uniformly Lipschitzian mappings in modular function spaces. Nonlinear Anal. 46, 267-278 (2001)

8. Dominguez-Benavides, T, Khamsi, MA, Samadi, S: Asymptotically regular mappings in modular function spaces. Sci. Math. Jpn. 53, 295-304 (2001)

9. Dominguez-Benavides, T, Khamsi, MA, Samadi, S: Asymptotically nonexpansive mappings in modular function spaces. J. Math. Anal. Appl. 265(2), 249-263 (2002)

10. Khamsi, MA, Kozlowski, WM: On asymptotic pointwise contractions in modular function spaces. Nonlinear Anal. 73 2957-2967 (2010)

11. Khamsi, MA, Kozlowski, WM: On asymptotic pointwise nonexpansive mappings in modular function spaces. J. Math. Anal. Appl. 380(2), 697-708 (2011)

12. Kozlowski, WM: On the existence of common fixed points for semigroups of nonlinear mappings in modular function spaces. Comment. Math. 51(1), 81-98 (2011)

13. Bin Dehaish, BA, Kozlowski, WM: Fixed point iterations processes for asymptotic pointwise nonexpansive mappings in modular function spaces. Fixed Point Theory Appl. 2012, 118 (2012)

14. Suzuki, T: The set of common fixed points of one-parameter nonexpansive semigroup of mappings is $F(T(1)) \cap F(T(\sqrt{2}))$. Proc. Am. Math. Soc. 134(3), 673-681 (2005)

15. Mann, WR: Mean value methods in iteration. Proc. Am. Math. Soc. 4, 506-510 (1953)

16. Krasnosel'skii, MA: Two remarks on the method of successive approximation. Usp. Mat. Nauk 10, $123-127$ (1955) (in Russian)

17. Ishikawa, S: Fixed points by a new iteration method. Proc. Am. Math. Soc. 44, 147-150 (1974)

18. Reich, S: Fixed point iterations of nonexpansive mappings. Pac. J. Math. 60(2), 195-198 (1975)

19. Reich, S: Weak convergence theorems for nonexpansive mappings in Banach spaces. J. Math. Anal. Appl. 67, 274-276 (1979)

20. Bose, SC: Weak convergence to the fixed point of an asymptotically nonexpansive map. Proc. Am. Math. Soc. 68, 305-308 (1978)

21. Passty, GB: Construction of fixed points for asymptotically nonexpansive mappings. Proc. Am. Math. Soc. 84, 212-216 (1982)

22. Gornicki, J: Weak convergence theorems for asymptotically nonexpansive mappings in uniformly convex Banach spaces. Comment. Math. Univ. Carol. 30, 249-252 (1989)

23. Schu, J: Iterative construction of fixed points of asymptotically nonexpansive mappings. J. Math. Anal. Appl. 158, 407-413 (1991) 
24. Schu, J: Weak and strong convergence to fixed points of asymptotically nonexpansive mappings. Bull. Aust. Math Soc. 43, 153-159 (1991)

25. $\mathrm{Xu}, \mathrm{H}-\mathrm{K}$ : Existence and convergence for fixed points of asymptotically nonexpansive type. Nonlinear Anal. 16 1139-1146 (1991)

26. Tan, K-K, Xu, H-K: An ergodic theorem for nonlinear semigroups of Lipschitzian mappings in Banach spaces. Nonlinear Anal. 19(9), 805-813 (1992)

27. Tan, K-K, Xu, H-K: Approximating fixed points of nonexpansive mappings by the Ishikawa iteration process. J. Math. Anal. Appl. 178, 301-308 (1993)

28. Bruck, R, Kuczumow, T, Reich, S: Convergence of iterates of asymptotically nonexpansive mappings in Banach spaces with the uniform Opial property. Colloq. Math. 65(2), 169-179 (1993)

29. Tan, K-K, Xu, H-K: Fixed point iteration processes for asymptotically nonexpansive mappings. Proc. Am. Math. Soc. $122,733-739$ (1994)

30. Rhoades, BE: Fixed point iterations for certain nonlinear mappings. J. Math. Anal. Appl. 183, 118-120 (1994)

31. Jung, JS, Kim, TH: Approximating fixed points of nonlinear mappings in Banach spaces. Ann. Univ. Mariae Curie-Skłodowska 51, 149-165 (1997)

32. Kaczor, W, Kuczumow, T, Reich, S: A mean ergodic theorem for nonlinear semigroups which are asymptotically nonexpansive in the intermediate sense. J. Math. Anal. Appl. 246, 1-27 (2000)

33. Kaczor, W, Kuczumow, T, Reich, S: A mean ergodic theorem for mappings which are asymptotically nonexpansive in the intermediate sense. Nonlinear Anal. 47, 2731-2742 (2001)

34. Garcia Falset, J, Kaczor, W, Kuczumow, T, Reich, S: Weak convergence theorems for asymptotically nonexpansive mappings and semigroups. Nonlinear Anal. 43, 377-401 (2001)

35. Noor, MA, Xu, B: Fixed point iterations for asymptotically nonexpansive mappings in Banach spaces. J. Math. Anal. Appl. 267, 444-453 (2002)

36. Khamsi, MA: On asymptotically nonexpansive mappings in hyperconvex metric spaces. Proc. Am. Math. Soc. 132 365-373 (2004)

37. Suzuki, T, Takahashi, W: Strong convergence of Mann's type sequences for one-parameter nonexpansive semigroups in general Banach spaces. J. Nonlinear Convex Anal. 5, 209-216 (2004)

38. Suzuki, T: Common fixed points of one-parameter nonexpansive semigroup. Bull. Lond. Math. Soc. 38, 1009-1018 (2006)

39. Hussain, N, Khamsi, MA: On asymptotic pointwise contractions in metric spaces. Nonlinear Anal. 71(10), 4423-4429 (2009)

40. Lewicki, G, Marino, G: On some algorithms in Banach spaces finding fixed points of nonlinear mappings. Nonlinear Anal. 71, 3964-3972 (2009)

41. Kozlowski, WM: Fixed point iteration processes for asymptotic pointwise nonexpansive mappings in Banach spaces. J. Math. Anal. Appl. 377(1), 43-52 (2011)

42. Kozlowski, WM: Common fixed points for semigroups of pointwise Lipschitzian mappings in Banach spaces. Bull. Aust. Math. Soc. 84, 353-361 (2011)

43. Kozlowski, WM: On the construction of common fixed points for semigroups of nonlinear mappings in uniformly convex and uniformly smooth Banach spaces. Comment. Math. 52(2), 113-136 (2012)

44. Kozlowski, WM: Pointwise Lipschitzian mappings in uniformly convex and uniformly smooth Banach spaces. Nonlinear Anal. 84, 50-60 (2013)

45. Kozlowski, WM, Sims, B: On the convergence of iteration processes for semigroups of nonlinear mappings in Banach spaces. In: Bailey, DH, Bauschke, HH, Borwein, P, Garvan, F, Thera, M, Vanderwerff, JD, Wolkowicz, H (eds.) Computational and Analytical Mathematics. In Honor of Jonathan Borwein's 60th Birthday. Springer Proceedings in Mathematics and Statistics, vol. 50. Springer, New York (2013)

46. Kozlowski, WM: On common fixed points of semigroups of mappings nonexpansive with respect to convex function modulars. J. Nonlinear Convex Anal. 15 (2014, in press)

47. Kozlowski, WM: Notes on modular function spaces I. Comment. Math. 28, 91-104 (1988)

48. Kozlowski, WM: Notes on modular function spaces II. Comment. Math. 28, 105-120 (1988)

49. Chistyakov, W: Modular metric spaces, l: basic concepts. Nonlinear Anal. 72(1), 1-14 (2010)

50. Chistyakov, W: Modular metric spaces, II: application to superposition operators. Nonlinear Anal. 72(1), 15-30 (2010)

51. Bruck, RE: A common fixed point theorem for a commuting family of nonexpansive mappings. Pac. J. Math. 53, 59-71 (1974)

52. Khamsi, MA: A convexity property in modular function spaces. Math. Jpn. 44(2), 269-279 (1996)

53. Chen, S: Geometry of Orlicz spaces. Diss. Math. 356, 1-204 (1996)

54. Kaminska, A: On uniform convexity of Orlicz spaces. Indag. Math. 44(1), 27-36 (1982)

55. Kozlowski, WM: On nonlinear differential equations in generalized Musielak-Orlicz spaces. Comment. Math. 53(2), 13-33 (2013)

56. Musielak, J: Orlicz Spaces and Modular Spaces. Lecture Notes in Mathematics, vol. 1034. Springer, Berlin (1983)

$10.1186 / 1687-1812-2014-4$

Cite this article as: Alsulami and Kozlowski: On the set of common fixed points of semigroups of nonlinear mappings in modular function spaces. Fixed Point Theory and Applications 2014, 2014:4 